\title{
GENERAL CONSTRUCTION AND CLASSIFICATION OF MANIPULATORS FOR UNDERWATER VEHICLES
}

Marek Dawidziuk, Adam Olejnik

Naval Academy, Department of Underwater Works Technology in Gdynia, Poland

\section{ABSTRACT}

The article presents the general construction of an underwater vehicle manipulator along with a discussion of the materials used in their construction. The types of drive systems used by the manipulator have been characterised, distinguishing their advantages and disadvantages. The functions of the manipulator are specified in relation to the activities performed by it. Moreover, the paper discusses the manipulator's degrees of freedom with the specification of the formula for their calculation. The basic types of end effectors are presented as well as an outline of the classification of manipulators in relation to the tasks carried out.

Keywords: marine engineering, submarines, underwater works technology, underwater vehicles manipulators.

ARTICLE INFO

PolHypRes 2018 Vol. 63 Issue 2 pp. 21 - 32

ISSN: $1734-7009$ eISSN: 2084-0535

Review article

DOI: 10.2478/phr-2018-0009

Pages: 12, figures: 6 , tables: 0

Submission date: $\mathbf{0 8 . 0 3 . 2 0 1 8} \mathrm{r}$.

page www of the periodical: www.phr.net.pl

Acceptance for print: $01.06 .2018 \mathrm{r}$.

Publisher

Polish Hyperbaric Medicine and Technology Society 


\section{INTRODUCTION}

The history of remotely operated vehicles (ROV) begins in the second half of the twentieth century. One of the first constructions was a vehicle from the French engineer Dimitri Rebikoff, specialising in underwater photography. In 1952 he built the first submersible scooter "Torpille" (French "torpedo"), which then served as a model for the construction of the first ROV called "Caniche" (French for "poodle"). This vehicle was created to carry out underwater research of sunken wrecks lying at depths that were inaccessible to divers [1]. It did not have any manipulative capacity and only enabled remote observation of the underwater situation by means of a TV camera mounted on it.

Already a year later, in 1953 an American inventor William E. Denny demonstrated the prototype of a remotely operated underwater vehicle - Archie (Fig. 1.a) and its twin structure - Buster [12.a]. These vehicles were equipped with the first manipulators that extended the possibilities of submersible vehicles with manipulative functions. The aim of the project was to create remotely controlled underwater vehicles for the exploration of cargo shipwrecks at greater depths than were inaccessible to divers.

The turn of the 1950s and 1960s saw the beginning of the dynamic development of underwater vehicles, and numerous prototypes and patents were registered. Already at that time it was noticed that supplying submersible vehicles with manipulators gave them operational features that had a major impact on the profitability of projects, their implementation and future development.

One of the driving forces behind the development of underwater vehicles equipped with manipulators in the 1960 s was an experimental torpedo recovery project for the US Navy. Figure 1.b. presents the prototype of the Solaris vehicle used in recovering training torpedoes. Solaris was also used to recover rocket-propelled missiles from the seabed following test firing and various underwater installations, as well as deploying explosives to destroy underwater objects [12.b].

The first underwater vehicles used in the US Navy equipped with extractors were the CURV-I and Monster (TONGS) vehicles. They appeared under the common code name XN-3 [1] and were used to secure the implementation of development torpedo and rocket programmes in order to recover test equipment. They were equipped with gripping devices which after grasping the object with a rope buoy or a recovery rope, enabled the extraction of the object from the bottom.

The industry associated with the exploration and extraction of new oil and gas deposits became another interested party in the design and development of this technology investing heavily in the development of underwater vehicles equipped with manipulators. The Shell Oil Company became aware of the need to invest in this new technology out of a necessity to carry out work at depths inaccessible to divers. At its request, the Hughes Aircraft Company constructed an underwater vehicle equipped with 4 MOBOT (mobile robot) manipulators, which were presented in 1963 [2] (Fig. 2.a).

The second half of the 1970s was a period of dynamic development of underwater vehicle manipulators in which specialised manipulators for specific tasks were developed - depending on the complexity of their design and their lifting capacity, these specialised manipulators were used for simple gripping and holding tasks as well as for more complex manipulation of objects. In one commercial underwater vehicle, Saab Scania's ORCA 1 (Fig. 2.b) from 1977, as many as three manipulators were used [3]. The main and most advanced manipulator on ORCA 1 was designed for manipulating objects and tools. The other two served as grippers, functioning as an aid to the main manipulator, and were responsible for immobilising the vehicle while working with hydrotechnical objects. It should be noted that already at that time it was proposed to use advanced manipulators for underwater works, which in their construction were similar to today's technical solutions.

In the initial stage of development of deep-sea vehicles, the manipulators often constituted their basic equipment. According to the US government agency NOAA (the National Oceanic and Atmospheric Administration) from the examined group of 104 deep water vehicles invented up to the end of the 1970s, as many as $33 \%$ were equipped with at least one manipulator [3]. Currently, there are many solutions of manipulators mounted on underwater vehicles, which, depending on the target functions performed, are characterised by a greater or lesser degree of constructional complexity. 


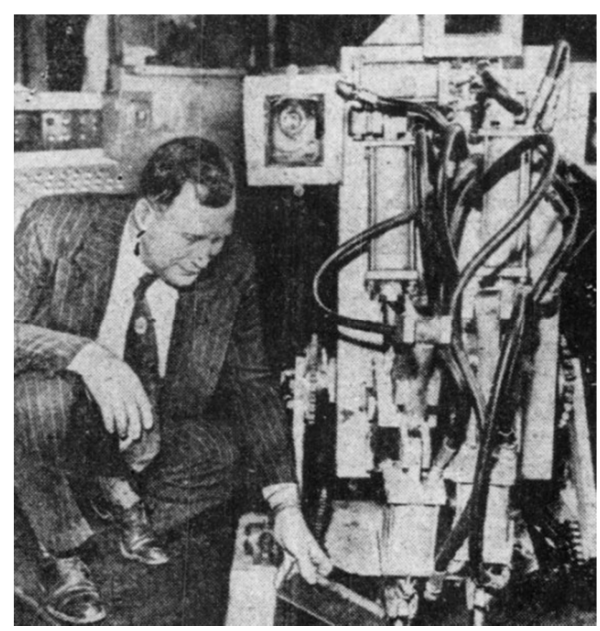

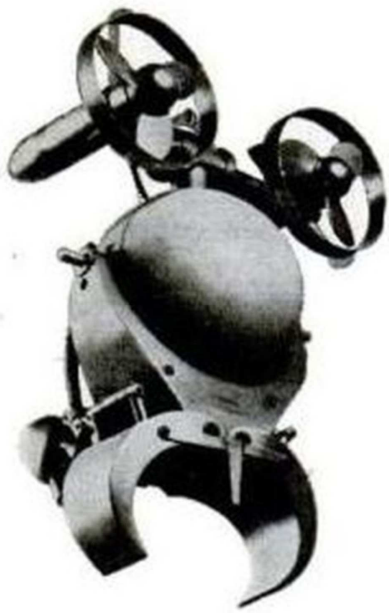

$\mathrm{B}$

Fig. 1 Prototypes of manipuled remotely operated underwater vehicles. A - Archie [4], B - Solaris [12.b].

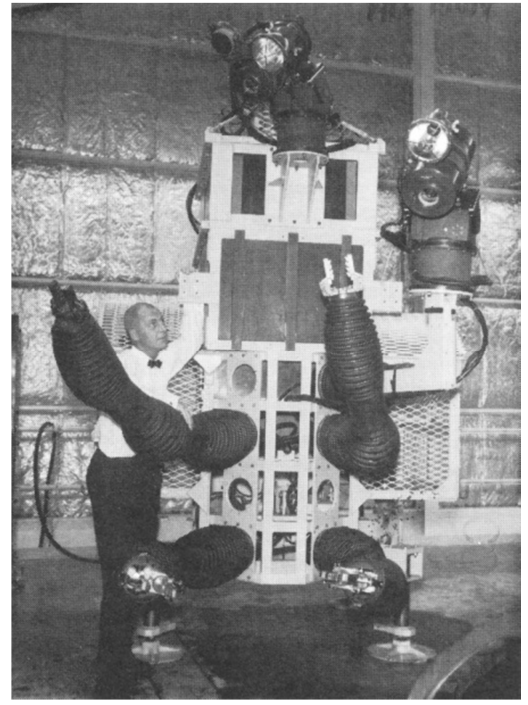

A

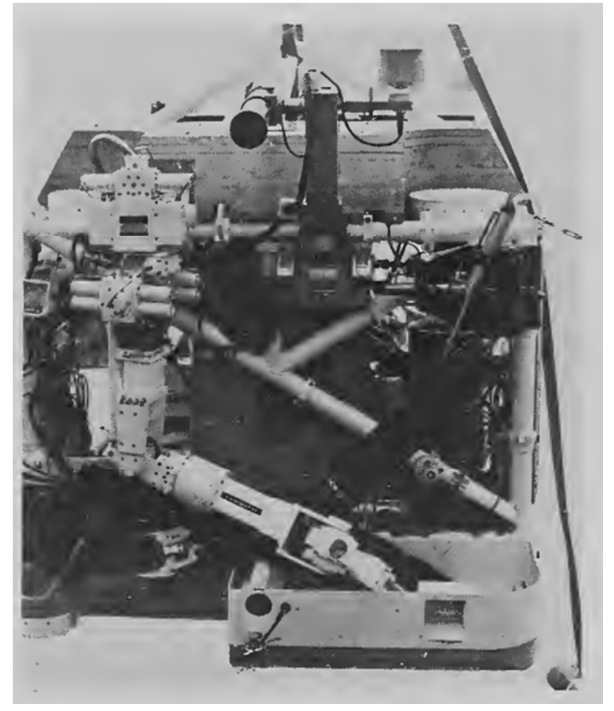

Fig. 2 Remotely operated underwater vehicles. A - prototype vehicle MOBOT [12.c], B-commercial vehicle ORCA 1 [3].

GENERAL

\section{STRUCTURE}

OF

\section{A MANIPULATOR FROM AN UNDERWATER VEHICLE}

An underwater vehicle manipulator is a remotely operated mechanical working arm mounted on the vehicle. According to the terminology provided by Zdanowicz [5], it can be classified as a group of industrial robot in the teleoperator class. Morawiecki and Knapczyk [6] use the term manipulator in reference to the equipment of an underwater robot, i.e. an underwater vehicle equipped with this type of device. The following functions of the ROV vehicle keypad can be distinguished in relation to the activity being performed:

- extension - performed by manipulator's arm in order to place the actuator in the working space of the device;

- $\quad$ manipulative - performed by the effector;

- $\quad$ sensory - realised by measurement devices. Most often these are measurements of resistance of movement of the manipulator arm members or measurement of gripper jaw pressure force. Measurements of the resistance of the arm members are most commonly used to simulate resistances in the control device, providing the operator with information on the operating conditions by means of force action of the control device elements in the operator's hand.

Basic manipulator constructions for gripping objects can be built from a gripper mounted on a simple extension arm (Figure 3.a). More advanced constructions consist of rigid members connected in series with joints enabling their relative movement (Fig. 3.b). In these advanced constructions, the first component part permanently connected to the ROV vehicle is at the same time the basis of the manipulator. The latter plays the role of an executive element - the end effector, later referred to as the effector. The construction of such a manipulator corresponds with the construction of an industrial robot with an open kinematic chain in which the effector is free and particular members are connected to a single preceding element. 


\section{DEGREES OF FREEDOM}

A set of two interconnected members creates a "kinematic pair" of a manipulator, these having a defined number of degrees of freedom (DOF). Underwater manipulators use kinematic pairs of 1 DOF, which allow changing only one of the six parameters describing the position in the three-dimensional space described by the Cartesian system (3 describe the position and 3 - orientation in relation to the system axis). In the vast majority of structures, we encounter kinematic systems designed exclusively on the basis of rotational pairs, enabling mutual change of the orientation of neighbouring members. Figure 4 shows a manipulator with seven degrees of freedom based on rotational kinematic pairs with the exception of the effector. There are also manipulators equipped with a single sliding pair.

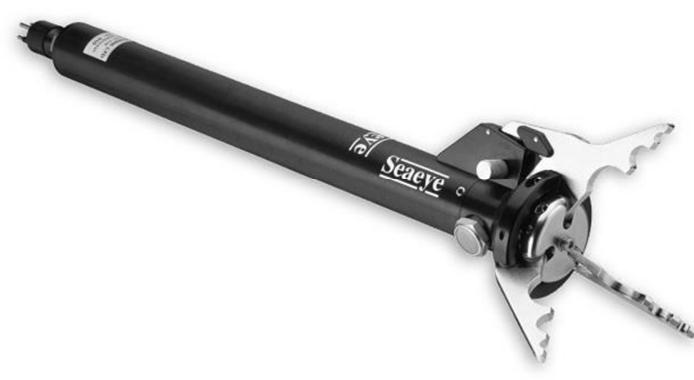

A
These are the manipulators: G500 by TitanRob [12i], and M2R offered by Profound Technology [12.j].

A single degree of freedom with respect to underwater manipulators is referred to as a single manipulator function $[7,8]$. These terms are often used interchangeably, as exemplified by the commercial market.

The manipulation possibilities of the manipulator mechanism are determined by two parameters: mobility and manoeuvrability. Acc. to Wojnarowski [9], a manipulator's mobility comes from the number of degrees of freedom of its kinematic chain in relation to the base, while manoeuvrability depends on the number of degrees of freedom of the manipulator mechanism - the robot with an immobilized base and its last link - the effector. Zdanowicz [5] also describes it as a number of independent drives that provide specific mechanism movement.

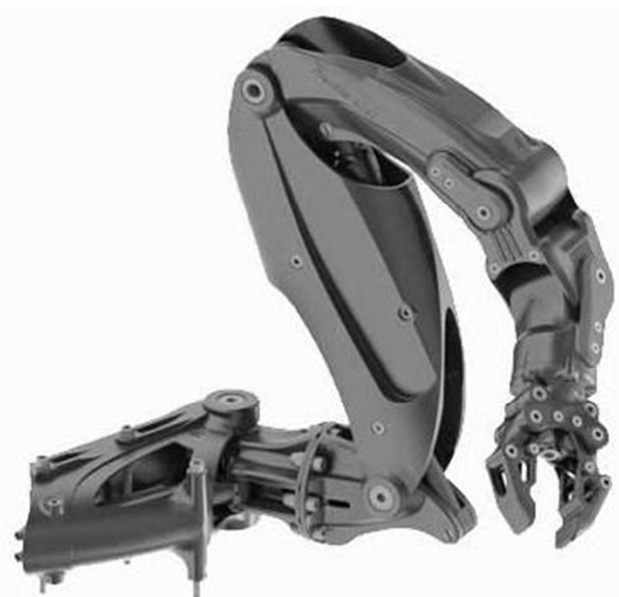

B

Fig. 3 Manipulators in underwater vehicles. A - GripStick manufactured by Saab SeaEye with a single function [12.e], B - 7-function M700 manufactured by TitanRob [12.f]

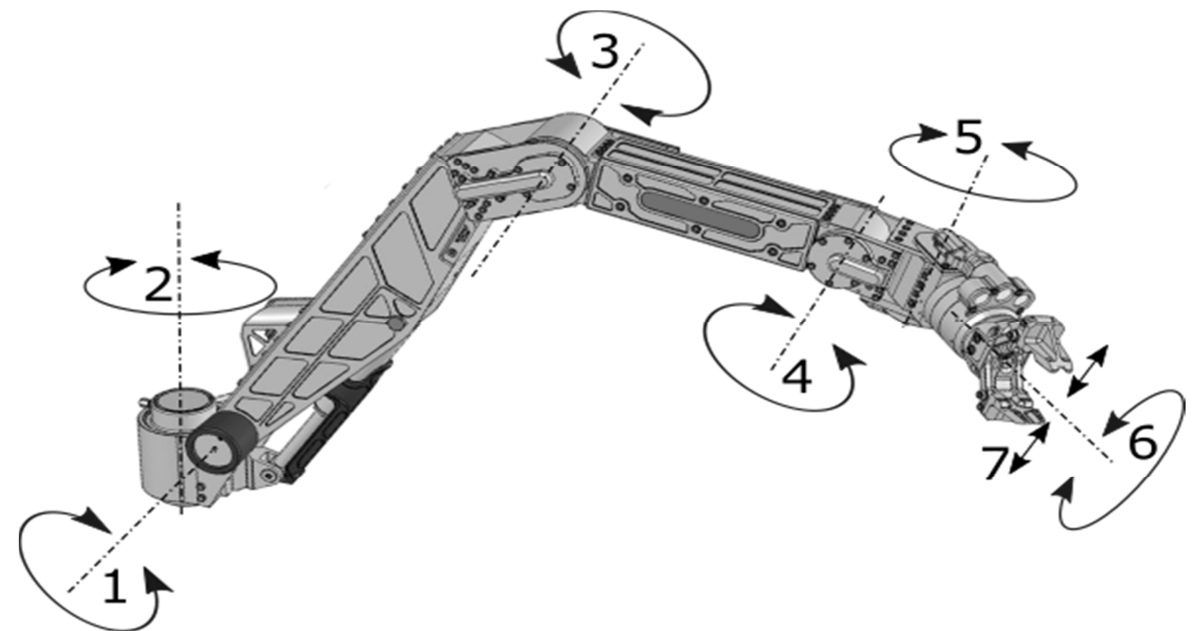

Fig. 4 Manipulator with 7 degrees of freedom. Based on [12.d]. 
The number of a particular manipulator's degrees of freedom can be calculated with the use of the following equation [5]:

$$
w=6 n-5 p_{5}-4 p_{4}-3 p_{3}-2 p_{2}-p_{1}=6 n-\sum_{i=1}^{5} i p_{i}
$$

where:

$$
\begin{aligned}
& n-\text { number of movable members in the manipulator, } \\
& p_{5}, p_{4}, \ldots, p_{1}-\text { number of kinematic pairs, each with } 1 \text { to } 5 \\
& \text { degrees of freedom (movement limitations). }
\end{aligned}
$$

Assuming that the manipulators of underwater vehicles are only provided with kinematic pairs of 1 DOF, the above formula can be reduced to:

$$
w=n
$$

Hence, the number of degrees of freedom of ROV manipulators is equal to the number of kinematic members. However, in the specialist literature dealing with issues of robotics $[5,6]$, the number of a manipulator's degrees of freedom is defined as the sum of the manipulator arm members without taking into account the effector's mobility. However, most manufacturers of ROV vehicle manipulators present it in their information brochures as the sum of the number of degrees of freedom of the manipulator arm with the number of degrees of freedom of the effector, which is confirmed by Christ and Wernli [7]. Moore [8] admits that manufacturers give varying degrees of freedom: with or without the consideration of the effector's mobility.

\section{MATERIALS USED IN MANIPULATOR CONSTRUCTION}

The manipulators of underwater vehicles are constructed using several main types of construction materials. These materials are resistant to corrosion resulting from atmospheric conditions and the aquatic environment. The basic materials include:

- $\quad$ stainless steel - usually a mixture of iron alloys with an admixture of chromium and nickel. It is a material of high mechanical strength and high specific gravity;

- anodised aluminium - aluminium alloy subjected to a special electrochemical process which forms an aluminium oxide layer on the aluminium surface, resulting in an increased hardness and corrosion resistance. It is characterised by a lower specific weight than stainless steel, and lower mechanical strength;

- titanium - materials made of titanium alloys are characterised by high mechanical strength. As regards their weight they take the position between materials made from aluminium alloys and stainless steel. They have the best strength to weight ratio in the class of working manipulators. The disadvantage of titanium alloys is their price;

- polymers - they are mainly used in manipulators for light work, dedicated to mini and compact classes of underwater vehicles. The most common polymers include:

$\checkmark$ high density polyethylene is a material resistant to water, salt solutions, certain acids, alcohols and gasoline. It is an easily workable and weldable material, with a weight lower than water;

$\checkmark$ polyoxymatylene from the group of thermoplastic polymers is characterised by good stiffness and strength at low own weight. It is characterised by natural lubricity.

\section{DRIVE SOURCES}

Two types of drive sources are used in modern manipulators of underwater vehicles: hydraulic and electric. The decision as to which manipulator drive is appropriate for any given situation results from its intended use and physical construction. Properties such as the load-carrying capacity of the drive, kinetic parameters of the manipulator, the number points of freedom and the accuracy of positioning are taking in to account when determining which drive to use.

The hydraulic drive is used in manipulators designed for the toughest jobs. This drive is characterised by high load-carrying capacity and rigidity, while at the same time not being sensitive to load changes. The rigidity of the drive units of the actuator structures allows their use as load-bearing elements of the device's construction. The operation of a hydraulic drive requires a lot of attention to the condition of the drive elements, the cleanliness of the working medium and proper condition of the hydraulic hose connections. Manipulators with hydraulic drives, in addition to actuators converting energy from the working medium into mechanical energy, require the use of auxiliary equipment to create pressure of the working medium, distribution, and to manage the intensity and pressure of its flow - all additional equipment increases the size and weight of the entire device. As shown by the market research, currently almost $70 \%$ of the manipulators offered for sale have a hydraulic drive.

The electric drive is mainly used in small manipulators with a small number of degrees of freedom. It is characterised by unchangeable work parameters, in particular the temperature-dependent parameters. This drive makes it possible to achieve high accuracy in the handling of manipulation elements, as well as the use of simple and cheap control systems.

Lack of a working medium and possible leaks means that there is no threat of environmental pollution, however, in electric manipulators adapted to work at greater depths, the empty spaces are filled with oils to increase their resistance to external pressure. The small physical dimensions of the control elements and the compact design of the drive elements enable their positioning in the manipulator frame, thus reducing the volume of the entire device. What has an unfavourable impact on the use of manipulators with an electric drive is the need to maintain a high degree of isolation of electrical components and their sensitivity to long-lasting overloads that may damage gearboxes and electric motors.

A separate group of manipulators are servohydraulic manipulators. The main drive of the kinematic members is in this case a hydraulic drive, while the electrical components are used to measure the mutual position of the manipulator members and the external forces acting on them. 


\section{THE END EFFECTORS OF UNDERWATER} MANIPULATORS

The end effector is a device placed on the last member of the arm. It is the main part of the manipulator, which is responsible for interaction with the working environment. The manipulator arm is responsible for placing the effector in the working space of the device, the task of the effector is to affect the environment. The effector's capabilities and functions depend on the purpose of the manipulator and the type of work performed. Sometimes they need to be specially designed devices, but most of the work can be done using standard constructions, among which the following types of effectors can be distinguished:

- grippers: the most common and useful effectors designed to manipulate objects. Their task is to capture objects and perform specific activities on them, such as changing their position, orientation or physical condition. In addition, grippers are used to operate tools specially designed for underwater vehicles. The principles of constructing the handles of such tools are presented in the standardisation document EN ISO 13628-8: 2006 [10]. Grippers consist of a drive unit, drive transmission and gripping ends. In constructions intended for heavier work, parallel and jaw grippers are used. In simple constructions, three-jaw grippers (tridents) are also used. Figure 5 shows different types of grippers. In some sources [8] one may find grippers divided into two categories, those of precision and forceful grippers. This classification results from the purpose of the manipulator. Work manipulators with a lifting capacity of up to $5 \mathrm{kN}$ equipped with grippers with a clamping force of $4 \div 6.4 \mathrm{kN}$ are ideal for heavy objects. They are not suitable for delicate and precise tasks. Practice shows that under water, the manipulator is not suitable for handling objects weighing less than $10 \%$ of its nominal lifting capacity [6]. Hence, in large ROVs sets of 2 manipulators with different grippers are used: precise (manipulative) and forceful for heavy work;

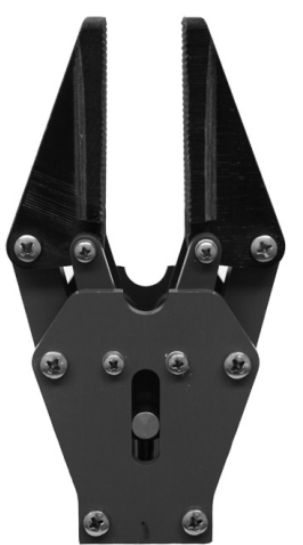

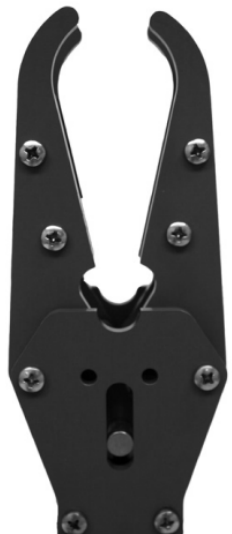

$\mathrm{B}$

Fig. 5 Gripping effectors [12.g]: A - parallel, B - jaw gripper, C - trident.

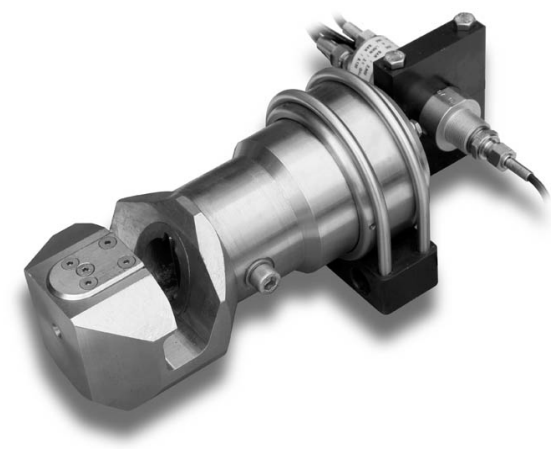

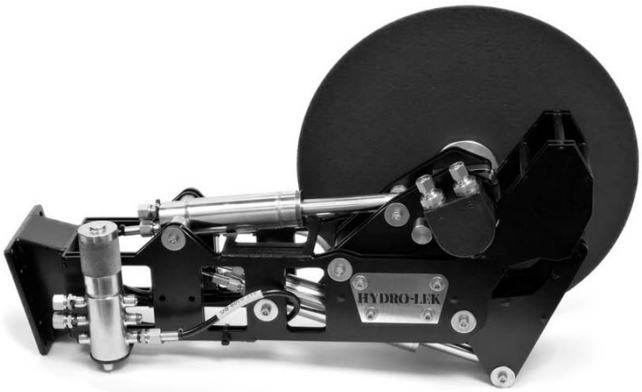

B

Fig. 6 Cutting effectors [12.h]: A - piston effector using a cutting blade, B - rotational (disc). 
- mechanical cutters: effectors provided with hardened blades for cutting cables, ropes and pipes (Fig. 6.a). These are devices with hydraulic drives in which the cutting element is set in plane motion. Another type of cutter is a rotary cutter (Fig. 6.b), in which the cutting element is a rotational grinding disc;

- rotational attachments: effectors that allow the performance of operations requiring the use of torque. The effector usually uses the torque generated by the "wrist" of the manipulator. These devices are used for screwing, drilling, cleaning elements using rotating brushes and valve adjustments. The EN ISO 13628-8: 2006 standard [10] presents the classification of endings and design requirements of such effectors depending on the torque transferred.

\section{THE CLASSIFICATION OF UNDERWATER} MANIPULATORS

1) The comparison of manipulators used in underwater vehicles and their unambiguous assignment to the group identifying them is problematic. The division of manipulators can be done by classifying them in terms of the type of work performed, the degree of complexity determined by the number of degrees of freedom or the type of drive used. Such a division can also be performed by considering the classification of the target carrier, i.e. the underwater vehicle itself, whose classification is also not a simple task [11].

2) The classification of manipulators in terms of their work may involve defining their task. There are numerous factors determining the purpose of a given manipulator. These include: the type of effector used, the number of degrees of freedom, arm length, capacity, working space and operating depth. When considering the tasks performed, the following groups of manipulators can be distinguished:

- manipulative - performing all kinds of work that require impact on the object mainly with the use of parallel and jaw gripping effectors (Figure 5). Usually, these are the most complicated manipulators with a large number of degrees of freedom with advanced controls;

- holding - intended to hold the object in order to immobilise it and perform operations using an additional manipulator. The effectors of such manipulators may take the form of vacuum grippers or suction cups;

- $\quad$ grippers - manipulators that handle large and heavy objects. These tasks are carried out by manipulators with a small number of degrees of freedom and a high gripping force of the effector. They often fulfil the task of keeping the vehicle in a stable position;

- $\quad$ auxiliary - manipulators dedicated to operating additional equipment such as additional camera or lighting. They are usually light constructions with a small number degrees of freedom;

- $\quad$ special - manipulators with special effectors specialised in strictly defined tasks. This group includes manipulators equipped with cleaning or rinsing effectors.

The classification of manipulators in terms of the number of degrees of freedom is the simplest division to be carried out, resulting directly from the manipulator's construction. We differentiate groups with the following number of degrees of freedom:

- $\quad$ from 1 to 2: simple manipulators used in small and medium ROVs. A single mobility is responsible for opening and closing the effector jaws. The second is usually responsible for the rotation of the effector around the longitudinal axis. These manipulators are most often found in low-cost vehicles and are built on the basis of electric drives. The low percentage of availability of these manipulators is explained by the fact that some ROV manufacturers offer simple manipulators of their own production without specifying their essential parameters;

- $\quad$ from 3 to 5 - manipulators designed for light and medium-duty underwater works, used as equipment on medium and large ROVs. They are characterised by the most favourable ratio of manipulation possibilities to their price.

- from 6 - manipulators with the most technically advanced constructions. The operability of such devices allows to manipulate objects in space in all the 6 points of freedom, as well as to bypass obstacles. In practice there are no manipulators with a greater number of points of freedom than 7 used in underwater vehicles, due to the significant complexity of the structure, and control problems that do not bring measurable benefits.

\section{Conclusions}

This article presented the general structure of ROV underwater vehicle manipulators. Attention was paid to the functions that the manipulator fulfils in relation to the performed activities. The concept of a manipulator's degrees of freedom (DOF) was discussed. Using the formula determining the number of degrees of freedom of the kinematic chain, a formula was developed for the number of degrees of freedom of manipulators used on underwater vehicles, where the important assumption is the use of 1-DOF kinematic elements in their construction.

The most popular construction materials used in the construction of manipulators were presented. The types of drive sources used were described, detailing their advantages and disadvantages.

Moreover, the concept of the end effector of the ROV vehicle manipulator was discussed. Three basic types of manipulator used for various purposes were distinguished and characterised. An outline of the classification of the manipulators in the function of tasks implemented by them and mobility was also demonstrated depending on the number of degrees of freedom of the manipulator's arm. Analyses highlighted that underwater manipulators offering more than 7 degrees of freedom are not in use. However, this number is sufficient to ensure optimal mobility of the manipulator arm. The limitation to applying more degrees of freedom is the problem of controlling such 
a manipulator, which will constitute the next stage of the analysis presented in a separate article.

\section{REFERENCES}

1. Olejnik A. The Development of the Technique of Underwater Remotely Operated Vehicles. Polish Hyperbaric Research 2009;3(28):7-21, ISSN 1734-7009

2. Meccano Magazine, Meccano Ltd. 1963; 2(48), 49;

3. Collective work edited by R. F. Busby. Remotely Operated Vehicles. U.S. National Oceanic and Atmospheric Administration - Office of Ocean Engineering, Washington D.C. 1979

4. The Ogden Standard-Examiner, Ogden Utah 17.05.1953; 2;

5. Zdanowicz R. The Basics of Robotics. Publ. Wydaw. Politechniki Śląskiej, Gliwice 2012: 29-83, ISBN 978-83-7335-922-2;

6. Collective work edited by Morawiecki A, Knapczyk J. The Basics of Robotics. Theory and Elements of Manipulators and Robots. $3^{\text {rd }}$ edition, WNT, Warsaw 1999: 412-439, ISBN 83-204-2331-7;

7. Christ R. D, Wernli Sr R. L. The ROV Manual. A User Guide for Remotely Operated Vehicles. Second edition 2014: 503-558, ISBN 978-0-08098288-5;

8. Moore S. W, Bohm H, Jensen V. Underwater Robotics. Science, Design \& Fabrication. MATE Center 2010: 529-572, ISBN 978-0-9841737-0-9;

9. Wojnarowski J, Nowak A. The Mechanics of Manipulators-Robots in Motor Description. Publ. Wyd. Politechniki Śląskiej, Gliwice 2007; 13-14, ISBN 978-83-7335-363-3.

10. European Standard. Petroleum and Natural Gas Industries - Design and Operation of Subsea Production Systems. Part 8: Remotely Operated Vehicle (ROV) Interfaces on Subsea Production Systems. EN ISO 13628-8:2006;

11. Jakus B, Olejnik A. The Analysis of Drive Systems in Unmanned Underwater Vehicles Towards Identifying the Method of Drive Transmission. Polish Hyperbaric Research 2017; 3(60), DOI 10.1515/phr-2017-00010

12. Internet sources:

http://cyberneticzoo.com/category/underwater-robotics/ - access in 06.2018;

http://cyberneticzoo.com/underwater-robotics/1960-solaris-mechanical-crab-jack-green-american/ - access in 06.2018;

http://cyberneticzoo.com/teleoperators/1964-unumo-universal-underwater-mobot-hughes-aircraft-american/ - access in 06.2018;

http://vistechnologies.com/?page id=661 - access in 06.2018;

http://www.saabseaeye.com/solutions/underwater-vehicles/falcon - access in 06.2018

http://www.titanrob.com/wp-content/uploads/2016/02/TRBT_SP003_M700-Specs_rev01.pdf - access in 06.2018;

http://www.videoray.com/homepage/new/options/rotating-manipulator.html\#!NewM̄Manip_Pro4 - access in 06.2018

http://hydro-lek.com/tooling.php - access in 06.2018;

http://www.titanrob.com/wp-content/uploads/2016/02/TRBT_SP002_G500-Specs_rev00.pdf - access in 06.2018;

http://www.profoundtechnology.net/manipulators/manipulator-m2r/ - access in 06.2018.

mgr inż. Marek Dawidziuk

Zakład Technologii Prac Podwodnych

Akademia Marynarki Wojennej

81-127 Gdynia, ul. Śmidowicza 69

tel. 48261262746

e-mail: m.dawidziuk@amw.gdynia.pl 